\title{
Changes in the anterior cornea during the early stages of severe myopia prior to and following LASIK, as detected by confocal microscopy
}

\author{
JINRONG ZHAO ${ }^{1}$, JINGUO YU ${ }^{2}$, LIU YANG $^{3}$, YANG LIU ${ }^{1}$ and SHAOZHEN ZHAO ${ }^{1}$ \\ ${ }^{1}$ Department of Refractive Optometry, Tianjin Medical University Eye Hospital, Tianjin $300384{ }^{2}$ Department of \\ Ophthalmology, Tianjin Medical University General Hospital, Tianjin 300052; ${ }^{3}$ Department of Ophthalmology, \\ Logistics University of People's Armed Police Forces Affiliated Hospital, Tianjin 300162, P.R. China
}

Received July 5, 2015; Accepted May 3, 2016

DOI: $10.3892 /$ etm.2017.4861

\begin{abstract}
The present study aimed to assess the microscopic changes of the epithelium and Bowman's layer under confocal microscopy during the early stages of severe myopia following laser in situ keratomileusis (LASIK). The study comprised 54 patients (54 eyes) with severe myopia and 65 patients (65 eyes) with low and moderate myopia, who underwent LASIK surgery at Tianjin Medical University Eye Hospital (Tianjin, China). In all patients, the epithelium, Bowman's layer and anterior stroma were examined prior to and 1, 7, 30,90 and 180 days after the surgery, using in vivo confocal microscopy. Six (6 eyes) of the 54 patients with severe myopia (11.1\%) exhibited corneal epithelial changes following the surgery, as examined by a slit lamp. Three patients (3 eyes) exhibited epithelial changes under confocal microscopy. In 40 eyes $(74.1 \%)$ with severe myopia, brown yellow changes were observed at the epithelial layer and in the central part of the cornea 6 months after the surgery; the same was observed in 35 eyes $(53.8 \%)$ with low and moderate myopia. Microfolds in the Bowman's layer of eyes with severe myopia were detected in 48 eyes (88.9\%), and in 54 eyes $(100.0 \%)$ in the anterior stroma following LASIK, whereas microfolds were observed in $51(78.5 \%)$ and 65 eyes (100\%), respectively, in patients with low to moderate myopia. In the severe myopia group, 54 eyes $(100 \%)$ exhibited nerve fibers below the epithelium that were detectable 1 day post-surgery; in 16 eyes (29.6\%), 1 or 2 sub-epithelial nerve fibers were detected 7 days after the surgery, and in 24 eyes (44.4\%), 1 or 2 sub-epithelial nerve fibers were observed 6 months after the surgery. In eyes with low and moderate myopia, the figures were 100, 38.5 and
\end{abstract}

Correspondence to: Dr Shaozhen Zhao, Department of Refractive Optometry, Tianjin Medical University Eye Hospital, 251 Fukang Road, Nankai, Tianjin 300384, P.R. China

E-mail: zhaosz1997@126.com

Key words: laser in situ keratomileusis, confocal microscopy, neurotrophic epitheliopathy
$64.8 \%$, respectively. The nerves of neither group returned to the pre-operative level. In conclusion, neurotrophic epitheliopathy, microfolds in Bowman's layer and anterior stroma were detected in the present patients with severe myopia of the cornea following LASIK, and the recovery of nerve fibers required an extended period of time.

\section{Introduction}

Laser in situ keratomileusis (LASIK) is a relatively novel technique for the correction of myopia which involves the creation of a corneal flap followed by ablation of the stroma using an excimer laser (1). The advent of in vivo confocal microscopy has provided the means of observing wound healing in the living cornea (2). Although numerous studies have investigated wound healing following $\operatorname{LASIK}(3,4)$, relatively few reports have addressed the epithelial changes associated with nerve damage following LASIK $(5,6)$. The cornea, which is comprised of sensory and autonomic nerve fibers, is one of the most richly innervated tissues in the body. These nerves have specific physiological roles, including the regulation of epithelial integrity, proliferation and wound healing $(7,8)$.

Corneal nerves are cut during the flap creation and stromal ablation stages of LASIK. One of the most common complications associated with LASIK is dry eye, followed by LASIK-induced neurotrophic epithelialpathy, which is secondary to surgically-induced neurotrophic keratopathy (9). The magnitude of corneal nerve damage is directly associated with the flap/ablation diameters, and the depth and degree of laser correction (10). Hence, high myopia would be expected to decrease post-operative corneal sensitivity and induce greater neurotrophic keratopathy. Therefore, the evaluation of damage in the epithelial and sub-basal nerve plexus may be an ideal way to assess the general nerve damage of the cornea. Previous studies have demonstrated that corneal nerves are degenerated during LASIK, and that regrowth of the sub-basal nerves is not complete a number of years after the surgery, although nerves in the hinge area are preserved $(11,12)$.

The present study was performed in Chinese patients with severe and low to moderate myopia, in order to analyze the 
epithelial and sub-basal nerve changes following LASIK. In vivo confocal microscopy was used to visualize the epithelium, sub-basal nerve plexus and anterior stroma in the central cornea prior to and at various time points following laser correction. This was performed in order to observe the difference between eyes with severe and low to moderate myopia, with regards to post-operative nerve damage, regrowth and surgical-induced neurotrophic epithelialpathy.

\section{Materials and methods}

Design. The present prospective, interventional cohort study was performed following ethical approval by the Tianjin Medical University Eye Hospital Ethics Committee, affiliated with Tianjin Medical University (Tianjin, China). Each patient provided written informed consent. In two groups of patients who underwent LASIK, patients with severe myopia and low to moderate myopia respectively, images of the cornea were captured using confocal microscopy, and data analysis was performed.

Patients. A total of 54 eyes of 54 patients (24 males, 24 eyes; 30 females, 30 eyes), with a diopter value between -10.00 and -17.00 diopter $(-13.00 \pm 2.1)$, were enrolled prospectively in a non-randomized fashion. A total of 65 eyes of 65 patients (30 males, 30 eyes; 35 females, 35 eyes), with a diopter value between -1.00 and -6.00 diopter $(-2.75 \pm 2.35 \mathrm{D})$, were enrolled as the control group. All patients were recruited from Tianjin Medical University Eye Hospital. Exclusion criteria included the wearing of contact lens, diabetes mellitus or other significant chronic disorders, glaucoma or ocular hypertension, the use of any ocular medications, and the use of systemic medications known to have adverse effects on the cornea. Confocal microscopy and a slit lamp were used to examine the respective corneas of all patients prior to and at various time points (1, 7, 30, 90 and 180 days) following LASIK. Uncorrected visual acuity was also evaluated.

In vivo confocal microscopy. Corneas were examined pre-operatively using a tandem scanning confocal microscope (Tandem Scanning Corporation, Reston, VA, USA). The specifications of this instrument and the methods used have previously been described by Patel et al (8). A drop of $0.2 \%$ carbomer gel (Vidisic; Mann Pharmaceuticals, Ltd., Berlin, Germany) was placed on the tip of the objective lens as an immersion fluid, and the lens was advanced until the solution made contact with the cornea. The objective lens of the confocal microscope was aligned with the center of the cornea by centering the light and dark rings of the epithelial image. Following alignment, the focal plane was scanned through the cornea at $72 \mathrm{~m} / \mathrm{sec}$ from the anterior to posterior, and digital images were stored on a computer workstation at 30 frames/sec. Each image represented a coronal section $475 \times 350 \mu \mathrm{m}$ (horizontal $\mathrm{x}$ vertical) and was separated from adjacent images by $2.4 \mu \mathrm{m}$. A scan through the full thickness of the cornea was completed in 7-9 sec. On each visit, the cornea was scanned between four and eight times. The objective lens and coupling medium were separated from the cornea between each scan. All scans were within the central $4 \mathrm{~mm}$ of the cornea, although they were not in the identical region each time. Therefore, the scans represented random, full-thickness samples of the central cornea. The central cornea of all patients were examined prior to the surgery, and 1 day, 1 week, and 1 , 3 and 6 months post-surgery.

Surgery and post-operative treatment. All the surgeries were performed by an experienced surgeon using a VISX S4 Excimer Laser (VISX, Santa Clara, CA, USA). Following topical anesthesia with oxybuprocaine hydrochloride eye drops (20 ml; 80 mg; Santen Pharmaceutical Co., Ltd., Osaka, Japan), a corneal flap (130 $\mu \mathrm{m}$ thickness; 8.5-9.0 mm diameter) was formed using an automatic microkeratome (M2; Moria Surgical, Antony, France). The flap was lifted and the central cornea underwent $6.5 \mathrm{~mm}$ laser ablation. Subsequently, the stromal bed was rinsed and the corneal flap was replaced. All patients were administered TobraDex eye drops $(5 \mathrm{ml}$; tobramycin, $15 \mathrm{mg}$; dexamethasone, $5 \mathrm{mg}$; Alcon-Couvreur NV, Puurs, Belgium) and a transparent eye patch. Following the surgery, patients were instructed not to rub their eyes and to avoid direct eye trauma. Post-operative treatment included antibiotic and steroidal eye drops, including $0.25 \%$ levofloxacin (5 ml; $24.4 \mathrm{mg}$; Santen Pharmaceutical Co., Ltd.) for 1 week and $0.1 \%$ fluorometholone (Allergan Pharmaceuticals, County Mayo, Ireland) eye drops for 4 weeks. In addition, $0.5 \%$ carboxymethylcellulose sodium eye drops (Allergan Pharmaceuticals) were used four times daily when punctuate defect epithelium changes occurred

Statistical analysis. The SPSS statistical software package (version 18; SPSS, Inc., Chicago, IL, USA) was used for all statistical analyses. Data are presented as the mean \pm standard deviation. $\mathrm{P}<0.05$ was considered to indicate a statistically significant difference.

\section{Results}

Neurotrophic epitheliopathy occurs following surgery. In the present study, six (6 eyes) of the 54 patients with severe myopia (11.1\%) exhibited a punctate epithelial defect following the surgery, as examined by a slit lamp; the majority of the lesions were on the lower part of the cornea. Three patients (3 eyes) experienced epithelial changes, observed using confocal microscopy. Among these, two patients (2 eyes) exhibited a basal cell layer with a circular or oval-shaped area of high reflectivity; for basal cells in this area, the cell boundaries were thickened and reflection was enhanced; the nuclei and cytoplasm underwent no obvious changes, however the corneal surface epithelial cell reflection was slightly increased (Fig. 1A). One patient exhibited enhanced surface epithelial cell reflection following surgery, in addition to the loss of some surface epithelial cells exhibited as dark, irregularly shaped areas. The wing cell and basal cell layers were not visible (Fig. 1B). Among the 6 patients who exhibited epithelial cell changes under the slit lamp, 3 also exhibited epithelial cell changes under a confocal microscope while the other 3 cases did not exhibit obvious morphological changes when examined by confocal microscopy. It can therefore be suggested that neurotrophic epitheliopathy is caused by LASIK. All presentations were resolved, as identified by examination using a slit lamp and confocal microscopy, following the use 

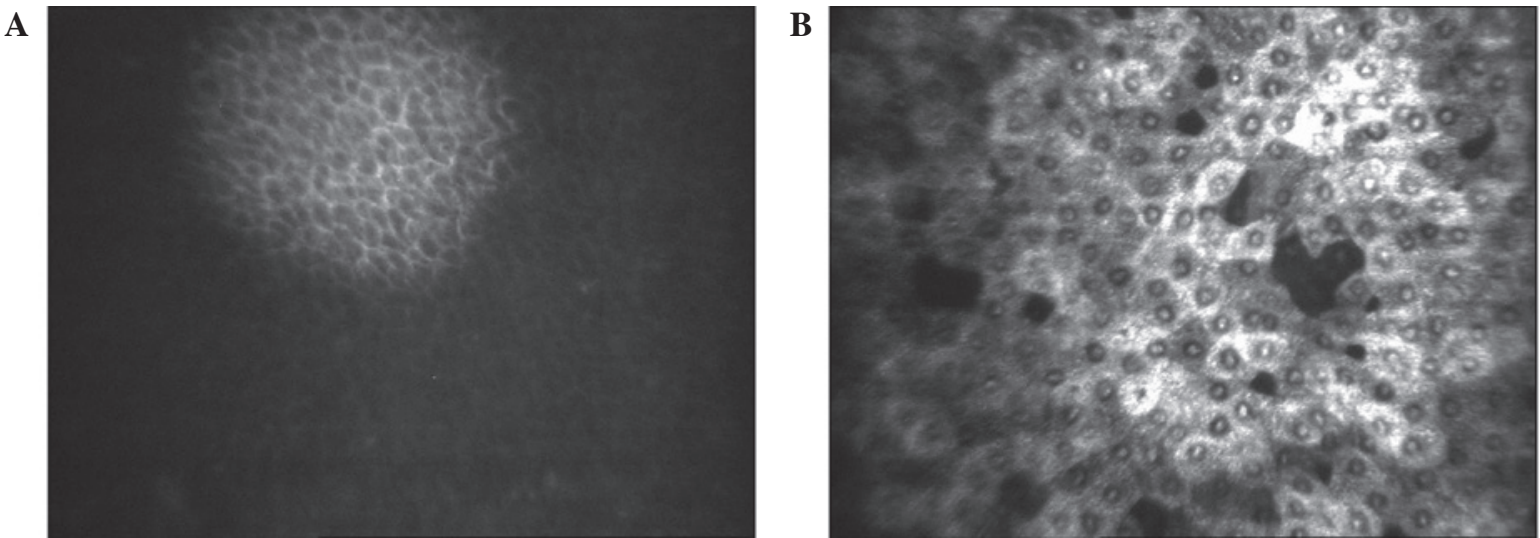

Figure 1. Neurotrophic epitheliopathy of a patient with severe myopia at 1 week post-surgery. (A) The basal cell layer was round with a high reflection area, and the basal cell boundary was thick with enhanced reflection. (B) Epithelial cell reflection was enhanced and some epithelial cells were lost. Magnification, x200.
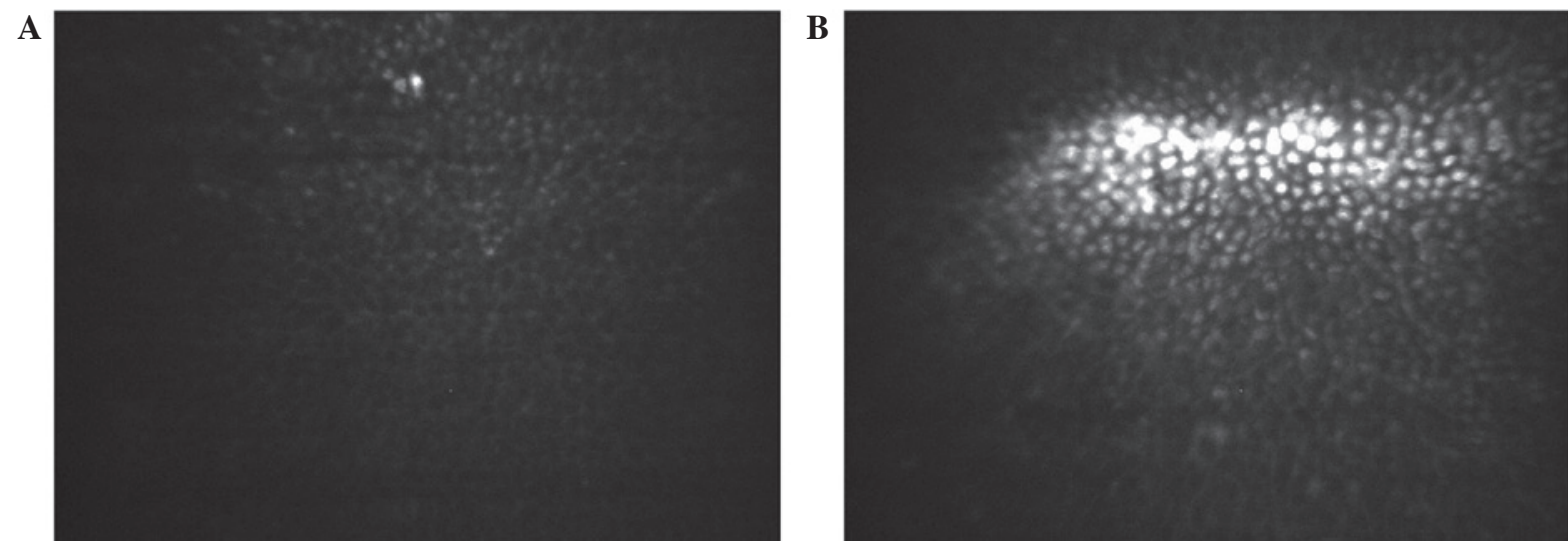

Figure 2. Epithelial layer debris of a patient with severe myopia. (A) At 1 week post-surgery, small, round, strongly light-reflecting dots converged in a cluster in the basal cell layer. These were deposits of ferric iron. (B) At 6 months post-surgery, the small, round, strong light-reflecting dots in the basal cell layer were increased in number and reflectivity compared with that at 1 week. Magnification, x200.

of carboxymethylcellulose sodium eye drops four times daily for 2 weeks. Only one patient with low to moderate myopia experienced corneal epithelial changes, as detected by slit lamp examination, but no obvious epithelial changes were detected by laser scanning confocal microscopy.

Epithelial layer deposits increase with time. In the severe myopia group, a total of 40 eyes (74.1\%) exhibited brown-yellow deposits at the epithelial layer of the central part of the cornea, as identified by slit lamp examination 6 months post-surgery. The deposits were nearly round or irregular, with a diameter of 1-2 mm. Confocal microscopy demonstrated small, round, strongly light-reflecting particles (diameter, 0.3-0.9 $\mu \mathrm{m}$ ), located in the basal cell layer near the Bowman's layer; the majority of the particles converged in a cluster. In two eyes (3.7\%) with severe myopia, deposits were detected at post-operative day 7 (Fig. 2A); however, the reflectivity was low and the areas of the deposits were quite small. The reflectivity and areas of the deposits increased gradually over the 6 months after surgery (Fig. 2B). The surrounding basal epithelium cells maintained normal morphology. In the low to moderate myopia group, 35 eyes (53.8\%) exhibited nearly round or irregularly shaped brown deposits at the central cornea, identified by slit lamp examination 6 months post-surgery. In the low to moderate myopia group, no eyes exhibited deposits at 7 days after surgery and only 1 eye exhibited low reflection, while at 3 months after surgery small, rounded, strongly light-reflecting particles were observed in the basal cell layer, although the areas were small and comparable with those observed in the severe myopia group 7 days after surgery. Confocal microscopy examination indicated that the number and areas of the reflective particles increased with time. They were more common in the eyes with high myopia.

Bowman's layer changes. In the severe myopia group, 48 eyes (88.9\%) exhibited wrinkles in the Bowman's layer one day after the surgery (Fig. 3), and 54 eyes (100\%) had wrinkles in the anterior stromal bed (Fig. 4). In the low to moderate myopia group, 51 eyes (78.5\%) demonstrated visible Bowman's layer folds, and 65 eyes (100\%) had anterior stromal wrinkles 1 day after the surgery. The wrinkles appeared as longitudinal or mesh-shaped dark regions, and studies have demonstrated that wrinkles gradually reduce with time (4). The patients of the current study were followed-up for 6 months after the surgery, and no reduction in wrinkles was observed.

Sub-basal nerve plexus analysis. In the severe myopia group, 54 eyes (100\%) developed sub-basal nerve plexus 1 day after 


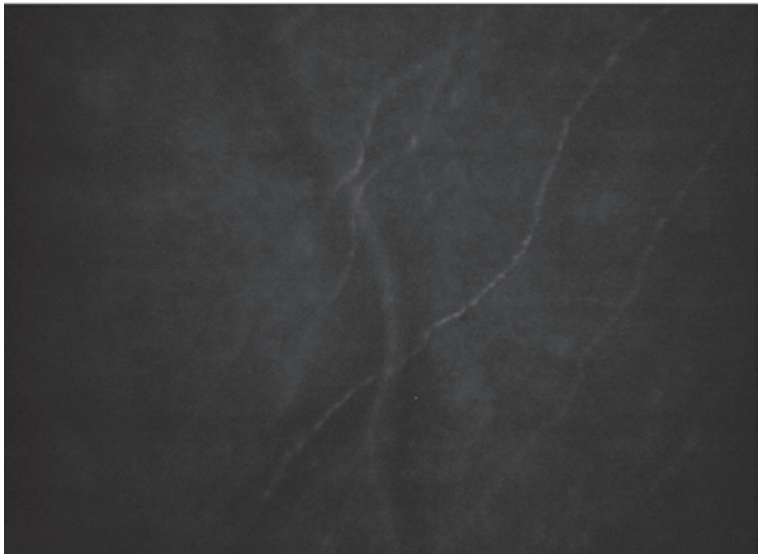

Figure 3. Bowman's layer folds of a patient with severe myopia. At 1 week post-surgery, the Bowman's layer nerve bent with the fold. Magnification, $\mathrm{x} 200$.

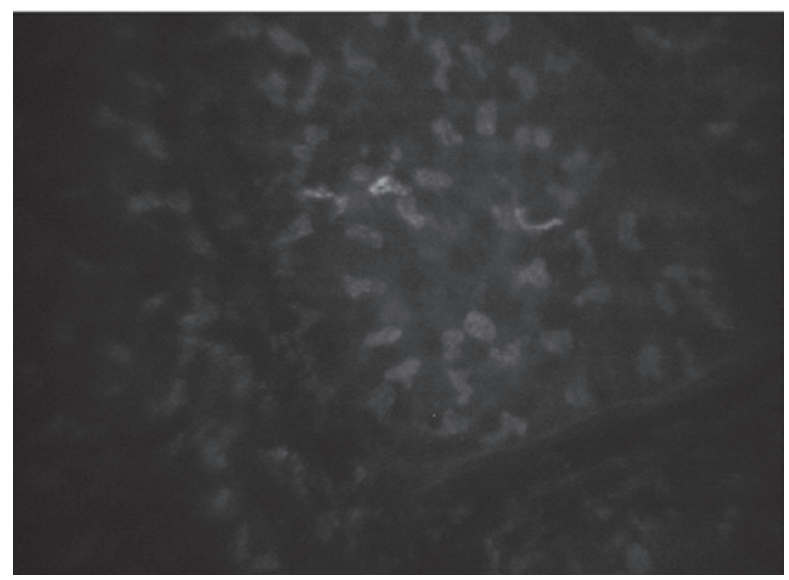

Figure 4. Anterior stroma wrinkles of a patient with severe myopia. At 1 week post-surgery, longitudinal or mesh shaped dark regions were observed in the stroma. Magnification, x200.

the surgery (Fig. 5A), whereas 16 eyes (29.6\%) developed one or two sub-epithelial nerve fibers 7 days after the surgery. In the remaining eyes, no sub-epithelial nerve fibers were observed. Up to 6 months after the surgery, 24 eyes (44.4\%) exhibited one or two sub-epithelial nerve fibers (Fig. 5B). In the low and moderate myopia group, the percentages of sub-basal nerves were $100,38.5$ and $64.8 \%$, respectively. In the two groups, the sub-epithelial nerve branches did not return to the pre-operative level.

\section{Discussion}

Corneal wound healing has become one of the most important factors affecting the stability and predictability of refractive surgery (13); in particular, it is important in high and severe myopia. It is possible to use confocal microscopy to dynamically observe changes in corneal tissue occurring in vivo at different time points following LASIK at a cytological level, and this may facilitate the observation of live corneal cell morphology and measurement of the number of cells, providing first hand, non-traumatic, objective and quantitative data for research purposes.
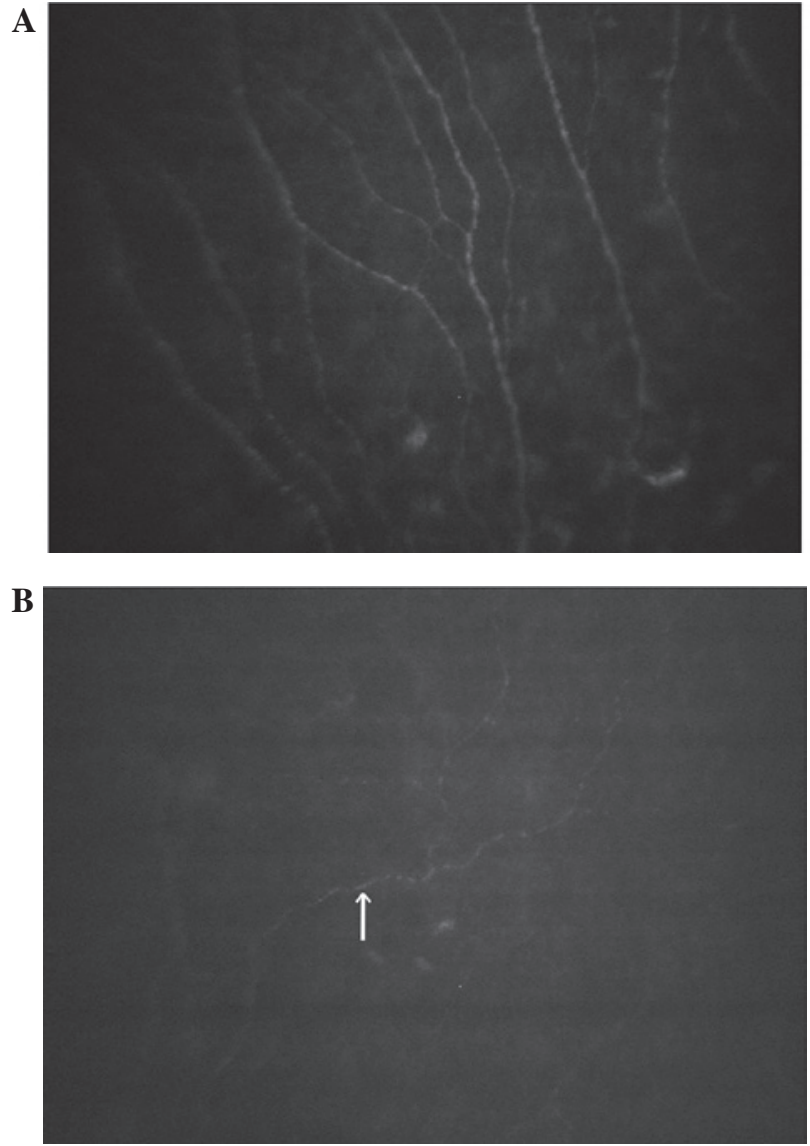

Figure 5. Sub-basal nerve of a severe myopia patient. (A) At 1 day post-surgery, the sub-basal nerve was unchanged compared with that prior to the surgery, whereas at (B) 1 week post-surgery, only 1 or 2 sub-epithelial nerve fibers were scarcely visible in the Bowman's layer (arrow). Magnification, x200.

The cornea, which is composed of sensory and autonomic nerve fibers, is one of the most richly innervated tissues in the body. These nerves have specific physiological roles, including regulating epithelial integrity, proliferation and wound healing $(7,8)$. Corneal nerves are cut during the flap creation and stromal ablation of LASIK. Due to the loss of neurological functions of epithelial cells, the corneal epithelium will change (5). The present study described 6 eyes of patients with severe myopia $(11.1 \%)$ with visible dotted epithelial lesions under slit lamp microscopy. Wilson (6) previously reported that the rate of epithelial complications from LASIK is $\sim 4 \%$. It has previously been demonstrated that the magnitude of corneal nerve damage is directly associated with the flap and ablation diameters, and the depth and degree of laser correction (10). Individuals who develop LASIK-induced neurotrophic epitheliopathy are typically asymptomatic, whereas a number of patients may have decreased visual quality and other visual discomforts, or abnormal sensations, which may be related to the corneal denervation effects. A number of these symptoms are self-limiting, and may be resolved 6 months after LASIK. These effects may remain in some individuals for a longer period of time, which is consistent with corneal nerve regeneration following LASIK (5). Post-operative dry eye and corneal nerve function loss may also have a role in neurotrophic epitheliopathy. This is consistent with corneal nerve regeneration following LASIK (14). 
When forming the flap and performing laser ablation during LASIK surgery, the nutritional function of cholinergic sensory nerve fibers on the corneal epithelium decreased as the nerves were damaged during surgery, resulting in a declined epithelial growth rate that may affect wound healing following LASIK (14). Even in the absence of trauma, neurological function loss can result in epithelial defects (15). In the present study, LASIK-induced neurotrophic epitheliopathy gradually disappeared and corneal nerve regeneration and recovery were induced over time. The associated pathological changes were generally mild and confined to the epithelial lesions.

The majority of patients develop epithelial deposits on the central corneal following LASIK. Strong reflective particles may also be visible in healthy cornea (15). These particles may be induced by the iron in tears, resulting in the abnormal deposition of iron from post-operative tears under the epithelium $(16,17)$. The probability of deposition on the epithelium is higher in eyes with high myopia following surgery, which has been confirmed by a previous study. This is associated with the corrective diopter (10). There is no consensus regarding whether deposits have the potential to affect vision $(11,12)$; in the present study, no reductions in vision were identified following the surgery.

The current study observed that 48 eyes (88.9\%) with severe myopia developed wrinkles in the Bowman's layer, and 54 eyes $(100 \%)$ developed wrinkles in the anterior stromal fold; whereas in eyes with low to moderate myopia, these phenomena were identified in $51(78.5 \%)$ and $65(100 \%)$ eyes, respectively. Bowman's layer and anterior stromal wrinkles, which present as longitudinal or grid-like dark areas, may be associated with the stretch of the flap. Laser cutting alters the corneal flap and the stromal bed compatibility following LASIK. Previous studies have demonstrated that wrinkles reduce gradually with the passage of time $(16,17)$. Linna et al (14) speculated that microfolds and foreign particles at the interface of the cornea may cause irregular astigmatism and a reduction of visual acuity, post-operatively. Since the cutting depth is deeper in high myopia, the microfolds are more evident in high myopia, as compared with low to moderate myopia. In the present study, microwrinkles in both groups decreased gradually with time. However, the long-term existence of microfolds may be associated with a decrease in contrast vision and night vision, or other symptoms (14). Holladay et al (18) studied the visual function of seven patients following bilateral LASIK surgery and observed that the post-operative contrast sensitivity and contrast sensitivity threshold were reduced. It was hypothesized that this may be associated with the flat cutting and irregular shape of the corneal surface following the surgery. The impact of microfolds on the visual quality of patients requires further study.

Corneal nerves come from the nasociliary branch of the ophthalmic division of the trigeminal nerve. Nerve fiber bundles enter the limbus of the cornea at the level of the middle and anterior stroma in a radial manner, and then travel parallel to the cornea surface. As these nerve bundles travel, they undergo division into smaller branches to innervate the anterior and mid stroma. They then travel between the Bowman's layer and basal epithelial cells, forming the sub-basal nerve plexus (19). As the formation of the corneal flap during LASIK cuts the nerve of the cornea, the corneal nerve requires a long period of time to recover. A previous study has reported that the sub-basal nerve is not fully restored 5 years after LASIK (12).

The results of the present study demonstrated that nerve recovery in patients with high myopia is slower than that in patients with low and moderate myopia. This may be associated with the deeper cutting depth required in patients with high myopia, which causes increased nerve damage. This may delay the recovery of patients with high myopia following surgery. Furthermore, due to the lack of corneal sensation, the patient is more likely to develop an infection in the cornea, which may cause increased damage to the patient's vision.

As the observation time in the current study was short, and the number of patients involved was small, there may be limitations to the results of the study. An increased number of patients and longer-term research is required in order to further investigate corneal epithelial and Bowman' layer changes in patients with severe myopia following LASIK. Further research may help to improve the post-operative results of LASIK.

\section{References}

1. Shortt AJ and Allan BD: Photorefractive keratectomy (PRK) versus laser-assisted in-situ keratomileusis (LASIK) for myopia. Cochrane Database Syst Rev 19: CD005135, 2006.

2. McLaren JW, Bourne WM, Maguire LJ and Patel SV: Changes in keratocyte density and visual function five years after laser in situ keratomileusis: Femtosecond laser versus mechanical microkeratome. Am J Ophthalmol 160: 163-170, 2015.

3. Cañadas P, de Benito-Llopis L, Hernández-Verdejo JL and Teus MA: Comparison of keratocyte density after femtosecond laser vs mechanical microkeratome from 3 months up to 5 years after LASIK. Graefes Arch Clin Exp Ophthalmol 251: 2171-2179, 2013.

4. Ramírez M, Hernández-Quintela E, Sánchez-Huerta V and Naranjo-Tackman R: Confocal microscopy of corneal flap microfolds after LASIK. J Refract Surg 22: 155-158, 2006.

5. Wilson SE and Ambrósio R: Laser in situ keratomileusis-induced neurotropic epitheliopathy. Am J Ophthalmol 132: 405-406, 2001.

6. Wilson SE: Laser in situ keratomileusis-induced (presumed) neurotropic epitheliopathy. Ophthalmology 108: 1082-1087, 2001.

7. Oliveira-Soto L and Efron N: Morphology of corneal nerves using confocal microscopy. Cornea 20: 374-384, 2001.

8. Patel DV and McGhee CN: In vivo confocal microscopy of human corneal nerves in health, in ocular and systemic disease and following corneal surgery: A review. $\mathrm{Br} \mathrm{J}$ Ophthalmol 93: 853-860, 2009

9. Ambrósio R Jr, Tervo T and Wilson SE: LASIK-associated dry eye and neurotrophic epitheliopathy: Pathophysiology and strategies for prevention and treatment. J Refract Surg 24: 396-407, 2008.

10. Lee BH, McLaren JW, Erie JC, Hodge DO and Bourne WM: Reinnervation in the cornea after LASIK. Invest Ophthalmol Vis Sci 43: 3660-3664, 2002.

11. Erie JC, McLaren JW, Hodge DO and Bourne WM: Recovery of corneal subbasal nerve density after PRK and LASIK. Am J Ophthalmol 140: 1059-1064, 2005.

12. Patel SV, McLaren JW, Kittleson KM and Bourne WM: Subbasal nerve density and corneal sensitivity after laser in situ keratomileusis: Femtosecond laser vs. mechanical microkeratome. Arch Ophthalmol 128: 1413-1419, 2010.

13. Li Y, Zhang X, Zhong LXY and Yin YM: Clinical use of in vivo confocal microscopy through focusing in corneal refractive surgery. J Refract Surg 22 (Suppl): S1041-S1046, 2006. 
14. Linna TU, Vesaluoma MH, Pérez-Santonja JJ, Petroll WM, Alió JL and Tervo TM: Effect of myopic LASIK on corneal sensitivity and morphology of subbasal nerves. Invest Ophthalmol Vis Sci 41: 393-397, 2000.

15. Loh A, Hadziahmetovic M and Dunaief JL: Iron homeostasis and iron disease. Biochim Biophys Acta 1790, 637-649, 2009.

16. Linna TU,Pérez-Santonja JJ, Tervo KM, Sakla HF, Alió y Sanz JL and Tervo TM: Recovery of corneal nerve morphology following laser in situ keratomileusis. Exp Eye Res 66: 755-763, 1998.

17. Latvala T, Barraquer-Coll C, Tervo K and Tervo T: Corneal wound healing and morphology after eximer laser in situ keratomileusis in human eyes. J Refract Surg 12: 677-683, 1996.
18. Holladay JT, Dudeja DR and Chang J: Functional vision and corneal changes after laser in situ keratomileusis determined by contrast sensitivity, glare testing and corneal topography. J Cataract Refract Surg 25: 663-669, 1999.

19. Mohamed-Noriega K, Riau AK, Lwin NC, Chaurasia SS, Tan DT and Mehta JS: Early corneal nerve damage and recovery following small incision lenticule extraction (SMILE) and laser in situ keratomileusis (LASIK). Invest Ophthalmol Vis Sci 55: 1823-1834, 2014 